\title{
Integrated glycomic analysis of ovarian cancer side population cells
}

\author{
Ran Zhao ${ }^{1 \dagger}$, Xiaoxia Liu ${ }^{2,5+}$, Yisheng Wang ${ }^{2,5}$, Xiaoxiang Jie ${ }^{4}$, Ruihuan Qin ${ }^{3}$, Wenjun Qin ${ }^{3}$, Mengyu Zhang ${ }^{2}$, \\ Haiyan Tai ${ }^{2}$, Caiting Yang ${ }^{3}$, Lili Li ${ }^{3}$, Peike Peng ${ }^{3}$, Miaomiao Shao ${ }^{3}$, Xingwang Zhang ${ }^{3}$, Hao Wu $^{3}$, Yuanyuan Ruan ${ }^{3}$, \\ Congjian $\mathrm{Xu}^{1,2,4,5^{*} \neq}$, Shifang Ren ${ }^{3^{*} \neq}$ and Jianxin $\mathrm{Gu}^{3}$
}

\begin{abstract}
Background: Ovarian cancer is the most lethal gynecological malignancy due to its frequent recurrence and drug resistance even after successful initial treatment. Accumulating scientific evidence indicates that subpopulations of cancer cells with stem cell-like properties, such as so-called side population (SP) cells, are primarily responsible for these recurrences. A better understanding of SP cells may provide new clues for detecting and targeting these cancer-initiating cells and ultimately help to eradicate cancer. Changes in glycosylation patterns are remarkable features of SP cells. Here, we isolated SP cells from ovarian cancer cell lines and analyzed their glycosylation patterns using multiple glycomic strategies.
\end{abstract}

Methods: Six high-grade serous ovarian cancer cell lines were used for SP cell isolation. Among them, HO8910 pm, which contained the highest proportion of SP cells, was used for glycomic analysis of SP cells. Cell lysate of SP cells and main population cells was applied to lectin microarray and mass spectrometry for glycan profiling. Differently expressed glycan structures were further verified by lectin blot, flow cytometry, and real-time PCR analysis of their relevant enzymes.

Results: Expression of core fucosylated N-glycan and tumor-associated Tn, T and sT antigens were increased in SP cells. By contrast, SP cells exhibited decreased hybrid glycan, a2,3-linked sialic glycan and multivalent sialyl-glycan.

Conclusions: Glycan structures, such as Tn, T, sT antigens, and core fucosylation may serve as biomarkers of ovarian cancer stem cells.

Keywords: Ovarian cancer stem cells, Glycomic, Biomarker, Tn antigen, T antigen, sT antigen

\section{Background}

Ovarian cancer is one of the most lethal gynecological malignancies [1]. The high mortality rate of ovarian cancer has been attributed both to late diagnosis due to lack of early symptoms and to recurrence accompanied by drug resistance [2-4]. Although $80 \%$ of patients are

\footnotetext{
*Correspondence: xucj@hotmail.com; renshifang@fudan.edu.cn

${ }^{\dagger}$ Ran Zhao and Xiaoxia Liu contributed equally to this article

${ }^{\ddagger}$ Congjian Xu and Shifang Ren contributed equally to this article

2 Obstetrics and Gynecology Hospital of Fudan University, 419 Fang-Xie

Road, Shanghai 200011, People's Republic of China

${ }^{3}$ Key Laboratory of Glycoconjugate Research Ministry of Public

Health, Department of Biochemistry and Molecular Biology, School

of Basic Medical Sciences, Fudan University, 138 Yi-Xueyuan Road,

Shanghai 200032, People's Republic of China

Full list of author information is available at the end of the article
}

initially responsive to optimal cytoreductive surgery plus platinum-based chemotherapy, recurrent cancer is inevitable in vast majority of cases. Along with recurrence, drug resistance develops, leading to death in $70 \%$ of ovarian cancer patients [5]. Thus, recurrence and drug resistance are main challenges in ovarian cancer treatment.

Accumulating scientific evidence suggests that tumors are driven by relatively rare and biologically distinct cancer-initiating cells, so-called cancer stem cells (CSCs), which are capable of self-renewal and further differentiation [6-8]. These cells are resistant to regular chemotherapy because they remain quiescent for a long time, proliferate slowly and excrete drugs easily $[7,8]$. Detecting and eliminating CSCs would prohibit relapse and improve survival of ovarian cancer patients [9]. 
Glycosylation is the most prevalent post-translational modification involved in many physiological and pathological processes. A large number of studies have demonstrated that glycans participate in cancer initiation [10], development [11-14], and drug resistance [15]. In addition, several reports have suggested that glycans and glycosyltransferases play important roles in CSC function. For example, increased fucosylation promotes invasive and metastatic properties of head and neck cancer stem cells [16]. $\beta 1,4-$ $N$-Acetylgalactosaminyltransferase (GalNAcT) III modulates the stemness of colon cancer via EGFR signaling pathway [17]. Glycans can also serve as potential CSC biomarkers. In combination with traditional biomarkers, glycans can be used to isolate more malignant CSCs. For example, anti-CD133 antibody, together with SSA (Sambucus sieboldiana) lectin, can identify CSCs of higher tumourigenicity in hepatocellular carcinoma [18]. Moreover, expression pattern of certain glycans on CSCs is distinct from that on normal stem cells, thus may improve the specificity of detecting and targeting CSCs [19]. Consequently, glycan biomarkers have more advantages with fewer side effects to harm the normal stem cells than general CSC markers. Glycomic analysis may provide comprehensive glycome profiles and potential biomarkers of CSCs. The specific glycans of CSCs have potential clinical value as targets in patients undergoing chemotherapy.

So far, little is known about glycosylation of ovarian CSCs. In this study, an integrated strategy using lectin array and mass spectrometry (MS) was employed to obtain the global glyco-information of ovarian cancer side population (SP) cells. Core fucosylated $\mathrm{N}$-glycan and tumor-associated $\mathrm{Tn}, \mathrm{T}$ and $\mathrm{sT}$ antigens were increased, while hybrid glycan, $\alpha 2,3$-linked sialic glycan and multivalent sialyl-glycan were decreased in SP cells. These differentially expressed glycans may serve as potential biomarkers of ovarian cancer SP cells.

\section{Methods}

\section{Cell culture}

The human high-grade serous ovarian cancer cell lines SKOV3 and HO8910 and their highly metastatic sublines SKOV3 ip and HO8910 pm were purchased from the cell bank of Shanghai Institute of Biochemistry and Cell Biology (China). The high-grade ovarian cancer cell line A2780 and its cisplatin-resistant subline A2780-cp were obtained from Obstetrics and Gynecology Hospital of Fudan University (China). All cell lines were routinely cultured in DMEM (Gibco Life Technologies, Carlsbad, CA, USA) supplemented with $10 \%$ foetal bovine serum (Gibco) and $100 \mathrm{U} / \mathrm{ml}$ penicillin/streptomycin (Gibco).

\section{Side population sorting}

SP sorting was performed as previously described [20]. Briefly, the fluorescent dye Hoechst 33342 (SigmaAldrich, St. Louis, MO, USA) was added to a cell suspension $\left(1 \times 10^{6}\right.$ cells $\left./ \mathrm{ml}\right)$ at a final concentration of $5 \mu \mathrm{g} /$ $\mathrm{ml}$. After incubation at $37{ }^{\circ} \mathrm{C}$ for $90 \mathrm{~min}$, the cells were treated with $2 \mu \mathrm{g} / \mathrm{ml}$ propidium iodide (PI) and sorted on a MoFlo XDP cytometer (Beckman Coulter, Brea, CA, USA). Cells treated with verapamil before Hoechst 33342 staining were used as negative control. Main population cells at the G0 stage were collected.

\section{Spheroid formation assay}

SP cells or MP cells were seeded at 2000 per well in 24-well Ultra Low plates (Corning, New York, NY, USA) in DMEM/F12 medium (Gibco) containing $20 \mathrm{ng} / \mathrm{ml}$ fibroblast growth factor, $20 \mathrm{ng} / \mathrm{ml}$ epidermal growth factor, $2 \%$ B27, insulin (R\&D Systems, Minneapolis, MN, USA) and $2 \mathrm{mg} / \mathrm{ml}$ heparin (Sigma-Aldrich) and cultured for 21 days.

\section{Cell cycle analysis}

After cell sorting, $5 \times 10^{5} \mathrm{SP}$ cells and MP cells were suspended in phosphate-buffered saline (PBS) buffer containing $50 \mu \mathrm{g} / \mathrm{ml}$ PI dividedly, incubated in the dark for $30 \mathrm{~min}$ at room temperature, and analyzed with a CyAn ADP flow cytometer (Beckman Coulter). Data were analyzed with ModFit LT for Windows version 3.1.

\section{Lectin microarray}

After sorting, SP cells and MP cells were washed several times with PBS and frozen at $-80^{\circ} \mathrm{C}$. For lectin microarray analysis, $1 \times 10^{5}$ cells were resuspended in $1 \mathrm{ml}$ of PBS-T (containing $1 \%$ TritonX-100), followed by sonication for $15 \mathrm{~min}$ and centrifugation at $14,000 \mathrm{~g}$ for $15 \mathrm{~min}$. The supernatant was recovered. Proteins were quantitated using a micro BCA kit (Thermo Scientific) and labelled with fluorescent dye Cy3 (Thermo Scientific). Samples with protein concentration of $250 \mathrm{ng} / \mathrm{ml}$ were applied to a LecChip (Glyco Technica) and incubated at $20^{\circ} \mathrm{C}$ for $16 \mathrm{~h}$. The chip was then scanned with a GlycoStation Reader 1200 (Glyco Technica) confocal scanner. Each lectin in LecChip has three replicates. To be normalized, intensity of each well in lectin microarray was divided by the mean of total 135 wells' intensity of the chip. We repeated lectin microarray analysis of SP and MP cells using independent samples to overcome biological bias.

\section{Cell lysis preparation for mass spectrometry analysis}

SP cells were rinsed with PBS. After washing, 2 \% SDS containing protease inhibitor cocktail (Roche Diagnostics, Roche Applied Science, Meylan, France) was used to 
lyse the cells at $100{ }^{\circ} \mathrm{C}$ for $15 \mathrm{~min}$. The lysate was then centrifuged at 14,000 $\mathrm{g}$ for $30 \mathrm{~min}$, and the supernatant was collected. The protein concentration in the supernatant was quantitated using a BCA kit (Thermo Scientific, San Jose, CA, USA).

\section{$\mathrm{N}$-Glycan release and purification}

For each cell line, $400 \mu \mathrm{g}$ of protein in $200 \mu \mathrm{l}$ of $2 \%$ SDS was added to $200 \mu \mathrm{l}$ of $8 \mathrm{M}$ urea (Sigma-Aldrich) containing dithiothreitol (Sigma-Aldrich) to achieve a final concentration of $10 \mathrm{mM}$. After heating at $56{ }^{\circ} \mathrm{C}$ for $20 \mathrm{~min}$, the samples were incubated in $40 \mathrm{mM}$ ammonium bicarbonate (Sigma-Aldrich) containing $25 \mathrm{mM}$ iodoacetamide (Sigma-Aldrich) for $30 \mathrm{~min}$ at $37^{\circ} \mathrm{C}$ in the dark. The sample was transferred to an ultrafiltration unit (Amicon Ultra-0.5, Ultracel-10 membrane; Millipore, Billerica, MA) and centrifuged at 14,000 $\mathrm{g}$ for $15 \mathrm{~min}$. A volume of $200 \mu \mathrm{l}$ of $40 \mathrm{mM} \mathrm{NH}_{4} \mathrm{HCO}_{3}$ was added to the ultrafiltration unit and centrifuged to wash the sample. Thereafter, $2 \mu \mathrm{l}$ of PNGase F (New England BioLab, Ipswich, $\mathrm{MA}$ ) in $200 \mu \mathrm{l}$ of $40 \mathrm{mM} \mathrm{NH} \mathrm{HCO}_{3}$ was added to the device and incubated with shaking for $24 \mathrm{~h}$ at $37{ }^{\circ} \mathrm{C}$. The ultrafiltration unit was transferred to a new collection tube and centrifuged at $14,000 \mathrm{~g}$ for $15 \mathrm{~min}$, and the filter membrane was washed with $200 \mu \mathrm{l}$ of $40 \mathrm{mM} \mathrm{NH}_{4} \mathrm{HCO}_{3}$ for three times. The solution in the collection tube was recovered and lyophilizedin a vacuum freeze dryer (Martin Christ $\mathrm{GmbH}$, Osterode, Germany). To remove sialic acids, each sample was reconstituted in $50 \mathrm{mM}$ ammonium acetate buffer ( $\mathrm{pH}$ 5.5) followed by desialylation with neuraminidase $(15 \mathrm{mU})$ from $V$. cholerae (Roche) (Sigma-Aldrich, St. Louis, MO) at $37{ }^{\circ} \mathrm{C}$ overnight. Subsequently, all samples were dried in a SpeedVac and redissolved in $50 \mu \mathrm{l}$ of water (with $0.1 \%$ TFA).

The N-glycans in solution were purified and desalted using a Porous Graphic Carbon Solid-Phase Extraction (PGC-SPE) as previously described [21]. The PGC-SPE microcolumn was a GELoader tip filled with porous graphic carbon powder. The microcolumn was prepared with 6 volumes of $0.1 \%(\mathrm{v} / \mathrm{v})$ trifluoroacetic acid (TFA) in $80 \%$ acetonitrile $(\mathrm{ACN}) / \mathrm{H}_{2} \mathrm{O}(\mathrm{v} / \mathrm{v})$ and equilibrated with water. The $\mathrm{N}$-glycan solution was passed through the microcolumn 5 times to ensure complete adsorption. The $\mathrm{N}$-glycans were eluted with $400 \mu \mathrm{l}$ of $0.05 \%(\mathrm{v} / \mathrm{v})$ TFA in $25 \%(\mathrm{v} / \mathrm{v}) \mathrm{ACN}$ and lyophilized.

\section{Mass spectrometry analysis}

N-Glycans were characterized by AXIMA Resonance MALDI QIT TOF MS (Shimadzu Corp, Kyoto, Japan). The lyophilized desialylated glycans were resuspended in $20 \mu \mathrm{l}$ of water. Each sample $(1 \mu \mathrm{l})$ was spotted on a MALDI target and dried in air at room temperature. To recrystallize the glycans, $1 \mu \mathrm{l}$ of $12.5 \mathrm{mg} / \mathrm{ml}$ 2,5-dihydroxybenzoic acid matrix (DHB, Sigma-Aldrich, Germany) dissolved in 50 \% ACN containing $0.1 \%$ TFA was added to the sample target. Each sample was spotted in five replicates. Glycan structures were analyzed using Glyco Workbench (http://code.google.com/p/ glycoworkbench/).

Launchpad software (Shimadzu Biotech, Kyoto, Japan) was used to acquire and process the MALDI MS data. The relative peak intensities were calculated as previously described [22, 23], that relative intensity of each type of $\mathrm{N}$-glycan was calculated by dividing the intensity of a given type of $\mathrm{N}$-glycan by sum of the total $25 \mathrm{~N}$-glycans intensity. Relative standard deviation (RSD) percentages based on relative intensity values were used to estimate the stability of mass spectrometry. Relative intensity of the five replicates of a certain $\mathrm{N}$-glycan were averaged and compared between SP and MP cells. $p<0.05$ were considered statistically significant. SP/MP ratios were calculated to express magnitude of changes. Cut-off of significant changes was set as $>1.5$ or $<0.67$ folds.

\section{Lectin blot}

The proteins isolated from SP cells and MP cells were analyzed by SDS-PAGE and lectin blot. In brief, samples were mixed with $5 \times$ loading buffer, boiled and separated by $10 \%$ SDS-polyacrylamide gel electrophoresis. The proteins in the gels were transferred to PVDF membranes (Millipore, Bedford, MA, USA). The PVDF membranes were blocked with TBST $(150 \mathrm{mM} \mathrm{NaCl}$, $10 \mathrm{mM}$ Tris- $\mathrm{HCl}, 0.05 \% \mathrm{v} / \mathrm{v}$ Tween $20, \mathrm{pH} 7.5)$ containing $5 \%$ bovine serum albumin for $1 \mathrm{~h}$ at room temperature and incubated with biotinylated Agaricus bisporus (ABA; Vector Laboratories, Burlingame, CA) or Vicia villosa lectin (VVA; Vector Laboratories) for $2 \mathrm{~h}$ at room temperature. After washing with TBST for 3 times, the membranes were incubated with horseradish peroxidase streptavidin (Vector Laboratories) for $30 \mathrm{~min}$ at room temperature. Signals were subsequently detected using an ECL assay kit.

\section{Total RNA extraction and quantitative real-time PCR analysis}

Total RNA was extracted with TRIzol ${ }^{\mathrm{TM}}$ reagent (Invitrogen, Carlsbad, CA, USA), and $2 \mu \mathrm{g}$ of RNA was used to synthesize cDNA using an RT Master Mix kit (Takara, Shiga, Japan). Real-time PCR analysis was performed using an ABI 7500 Fast Real-time PCR system (Applied Biosystems, Switzerland) for 40 cycles $\left(15 \mathrm{~s}\right.$ at $95{ }^{\circ} \mathrm{C}$, $30 \mathrm{~s}$ at $60{ }^{\circ} \mathrm{C}$ ). A $2-\mu \mathrm{l}$ aliquot of cDNA was amplified in $20 \mu$ l system using the SYBR-green Premix Real-time PCR kit (Takara) system according to the manufacturer's instruction. The primer sequences were as follows: GAPDH, GTCAAGGCTGAGAACGGGAA (forward) 
and AAATGAGCCCCAGCCTTCTC (reverse); Oct4, GACAACAATGAAAATCTTCAGGAGA (forward) and TTCTGGCGCCGGTTACAGAACCA (reverse); Nanog, CAAAGGCAAACAACCCACTT (forward) and TCTGC TGGAGGCTGAGGTAT (reverse); Fut8, TCTTCATCCC CGTCCTCCA (forward) and GAGACACCCACCACA CTGCA (reverse); C1 galt1, CATCCCTTTGTGCCAGAA CACC (forward) and GCAAGATCAGAGCAGCAACC AG (reverse); St3gal1, GGGCAGACAGCAAAGGGAA (forward) and GGCCGTCACGTTAGACTCAAA (reverse).

\section{Flow cytometry}

After sorting, the SP cells and MP cells were cultured for 5 weeks in the previously mentioned medium in 6-well Ultra Low plates (Corning). The spheres were divided into single cells, stained with fluorescent-labelled ABA and VVA (Vector Laboratories) for $15 \mathrm{~min}$ at room temperature, and detected using CyAn ADP (Beckman Coulter).

\section{Statistical analyses}

Means of continuous data were compared using Student's $t$ test by SPSS software (version 16.0), $p<0.05$ was considered statistically significant. The results shown in figures were expressed as mean \pm SD.

\section{Results}

\section{Ovarian cancer SP cells exhibit stem cell-like properties}

To enrich SP cells, we analyzed six serous ovarian cancer cell lines, including SKOV3, HO8910 and their highly metastatic derivatives SKOV3 ip and HO8910 pm, as well as A2780 and its drug-resistant derivative A2780 cp. HO8910 pm cells exhibited $4.63 \%$ Hoechst low staining SP cells, as previously reported [20] (Fig. 1a). Other cell lines exhibited only small amounts of SP cells, which were inadequate for glycoprofiling. Thus, we chose HO8910 pm cells for further studies.

Compared with the main population (MP) cells, SP cells exhibited higher self-renewal capacity. After plating in ultra-low attachment plates for 3 weeks, the SP cells formed significantly larger numbers of spheroids than MP cells. Moreover, the spheroids of SP cells were much larger in size (Fig. 1b, c). To further characterize the stem cell-like features of SP cells, we analyzed the expression of stem genes using real-time PCR. Both Oct- 4 and Nanog were over-expressed in SP cells (Fig. 1d). CSCs are considered to reside in a stable quiescent state in the G0 state of the cell cycle and are therefore resistant to chemotherapies [7]. We also examined the cell cycle of SP cells: nearly $97.5 \%$ were arrested in the G0/G1 state (Fig. 1e), whereas only $66.17 \%$ of unsorted HO8910 pm cells were in the G1 state.

\section{Lectin array analysis of SP cells and MP cells}

To obtain more comprehensive information about the global glycosylation of SP cells, lectin array including 45 lectins were analyzed. Signal intensity of 9 lectins were significantly different between SP and MP cells in both of the two experiments (Fig. 2; Table 1).

Among O-glycan binding lectins, signal intensity of ABA, PNA, VVA, SBA, GSL-IA 4 , and GSL-IB $_{4}$ were higher in SP cells, indicating a higher expression of Tn (GalNAc- $\alpha$-Ser/Thr), T (Galß1-3 GalNAc- $\alpha-S e r / T h r)$, and sT (sialyl-T) antigens in these cells. Among these lectins, intensities of ABA, VVA, which recognize T, sT and Tn antigens, were the highest in two experiments, respec-

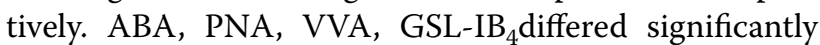
in the two experiments. In contrast, SP cells exhibited a significantly lower intensities for (Gal $\beta 1-4$ GlcNAc) (poly LacNAc) (LEL) and multivalent sialyl glycan binders (WGA).

Among N-glycans, SP cells showed significantly stronger intensity of fucosylation binder AAL, which recognizes the core fucose (Fuc) and Lewis X structure. A higher intensity for AOL, which recognizes both core Fuc and Fuc $\alpha 1-2$ (galactoside $\beta 1-4) n$-acetylglucosamine (Fuc $\alpha 1-2($ Gal $\beta 1-4)$ GlcNAc), was also observed in SP cells. However, SP cells exhibited relatively weak intensity to UEA-I, which recognizes Fuc $\alpha 1-2(\mathrm{Gal} \beta 1-4)$ GlcNAc. These results together indicated that core fucosylation glycans were increased in SP cells. In addition, the signal for $\alpha 2-3$ linked sialic acids (Sia) binders (MAL) was significantly decreased in SP cells.

MS analysis of the N-glycan profiles of SP cells and MP cells To obtain more detailed information about the N-glycan structures and their relative intensities, we compared $\mathrm{N}$-glycan profile of the whole proteome of SP cells and MP cells using MS. Twenty-five N-glycans were detected of both cell populations, and were shown in Fig. 3. Statistical analysis was conducted to determine the quantitative differences in glycans between SP and MP cells. A comparison based on different classes (high mannose, hybrid, and complex) were shown in Fig. 4. The relative intensities of each corresponding structure were analyzed by the method above and presented in Table 2 .

We observed that the proportion of high mannose type glycan was nearly identical in SP cells $(77.21 \%)$ and MP cells $(77.90 \%)$. Signal of $m / z 2067.9$ was significantly higher $(p<0.01$, fold change $>1.5)$ in SP cells. In addition, the proportion of hybrid type $\mathrm{N}$-glycan was obviously decreased in SP cells as compared with MP cells (10.44 vs. $12.30 \%)$. Most hybrid N-glycans were reduced in SP cells, 1460.8 was significantly decreased $(p<0.01$, fold change $<0.67)$ in SP cells. However, the percentage 


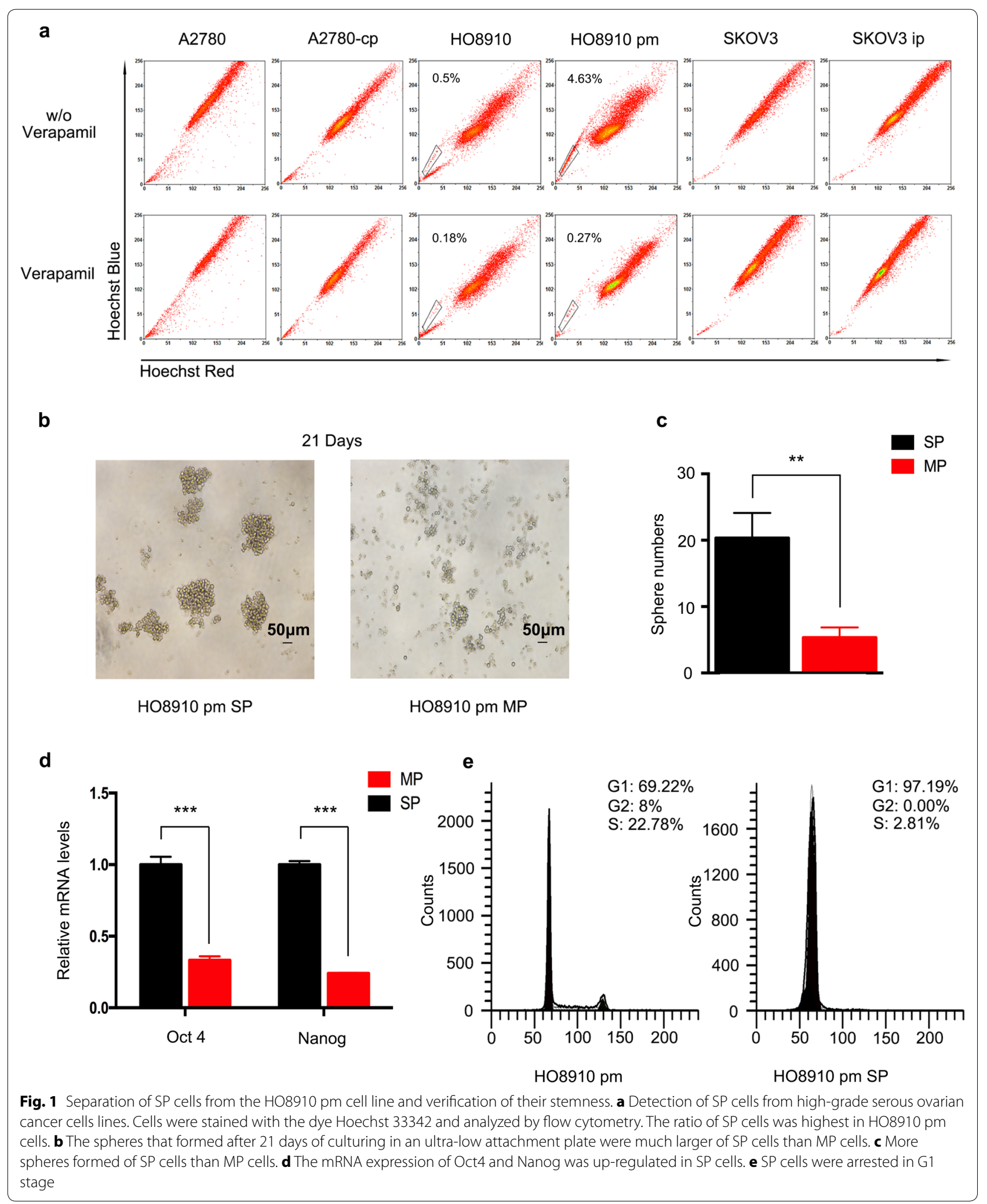


a

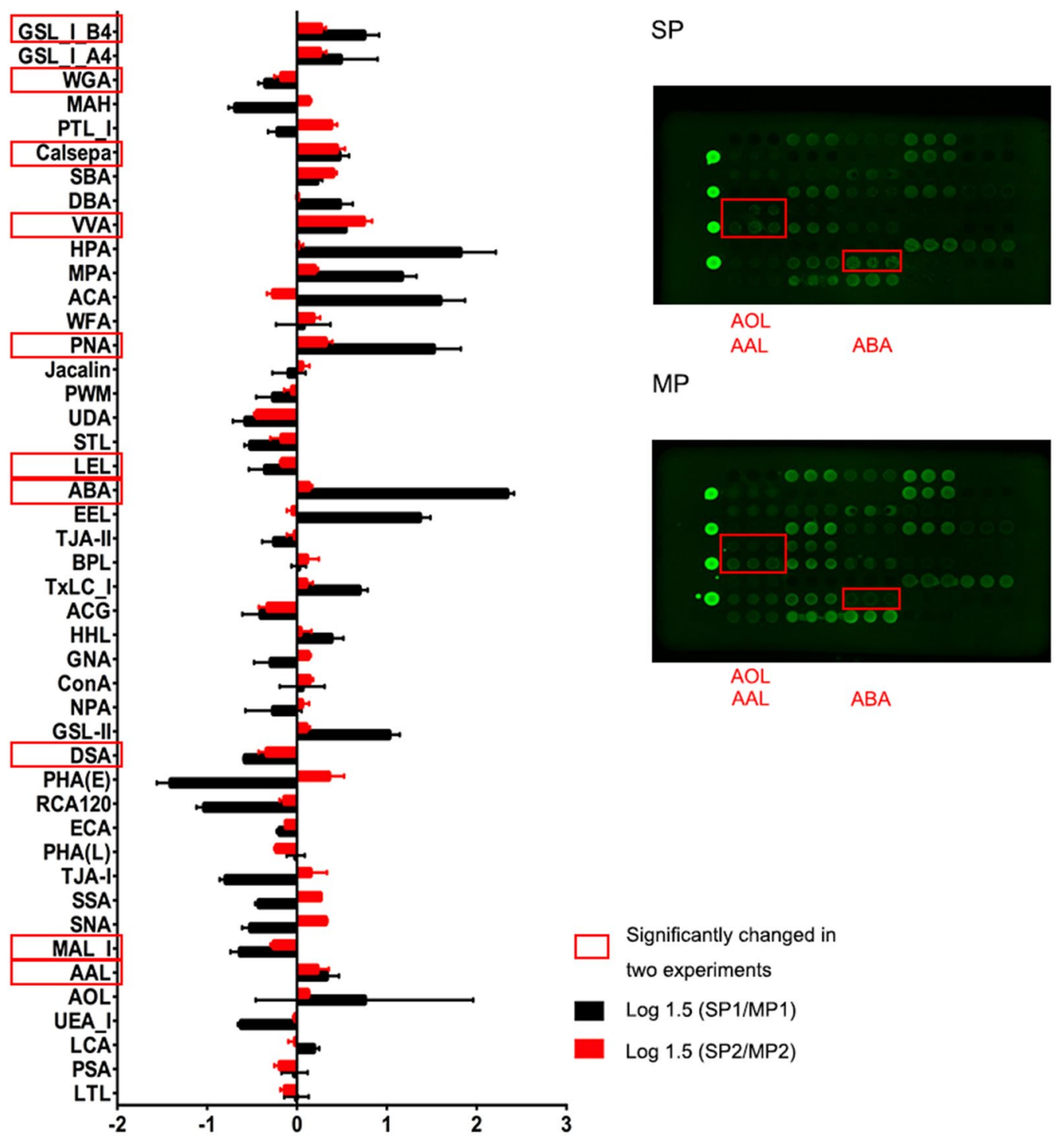

$\log 1.5(\mathrm{SP} / \mathrm{MP})$ b

SP

MP

Fig. 2 Lectin array analysis of SP cells and MP cells. a Relative intensities of 45 lectins in SP cells compared with MP cells for two experiments. b Fluorescence intensities of SP cells (up) and MP cells (down)

of complex N-glycans ranging from $\mathrm{m} / z 1200$ to $\mathrm{m} / z$ 2400 was slightly increased in SP cells (12.35 vs. 9.79\%). Among complex N-glycans, core fucosylated N-glycans, catalyzed by fucosyltransferase-8 (FUT8), were also higher in SP cells (4.64 vs. $3.42 \%$ ), and $m / z 1485.6$ and $m / z 1688.7$ were also significantly higher $(p<0.01$, fold change $>1.5$ ). These results correlated well with the lectin array results (Fig. 2). Moreover, real-time PCR was performed to further verify that SP cells expressed a higher level of FUT8-encoding gene Fut8 at mRNA level.
SP cells exhibit higher signal intensity for ABA and VVA

Lectin array analysis indicated that SP cells expressed more tumor-associated antigens which strongly bound to the lectins ABA and VVA. Among all 45 lectins, ABA, which can recognize both $\mathrm{T}$ and $\mathrm{sT}$ antigens, exhibited the strongest intensity for SP cells. VVA, which can recognize Tn antigen, also displayed significantly higher intensity for SP cells. Hence, we performed lectin blots to compare recognition of ABA and VVA between SP and MP cells. SP cells exhibited higher intensity for 
Table 1 Variation of glycans determined by lectin microarray analysis of SP cells and MP cells

\begin{tabular}{|c|c|c|c|c|c|c|c|c|}
\hline No. & Lectin & Preferred glycan structure (terminal epitope) & $\mathrm{SP} 1 \pm \mathrm{SD}$ & MP1 \pm SD & SP1/MP1 & $\mathrm{SP} 2 \pm \mathrm{SD}$ & $\mathrm{MP} 2 \pm \mathrm{SD}$ & SP2/MP2 \\
\hline 1 & LTL & $\begin{array}{l}\text { Fuc a1-3(Gal } \beta 1-4) \text { GlcNAc (Lex), Fuc a1-2(Gal } \beta 1-4) \\
\text { GlcNAc }\end{array}$ & $0.40 \pm 0.02$ & $0.40 \pm 0.02$ & 1.00 & $0.42 \pm 0.02$ & $0.45 \pm 0.02$ & 0.95 \\
\hline 2 & PSA & Fuc a1-6GlcNAc, a-D-Glc, a-D-Man & $0.78 \pm 0.01$ & $0.79 \pm 0.06$ & 0.99 & $0.82 \pm 0.04$ & $0.88 \pm 0.02$ & 0.93 \\
\hline 3 & LCA & Fuc a1-6GlcNAc, a-D-Glc, a-D-Man & $0.91 \pm 0.07$ & $0.84 \pm 0.06$ & 1.08 & $0.89 \pm 0.01$ & $0.89 \pm 0.03$ & 0.99 \\
\hline 4 & UEA-I & Fuc a1-2(Gal $\beta 1-4)$ GlcNAc & $0.41 \pm 0.02$ & $0.53 \pm 0.03$ & $0.78^{* *}$ & $0.60 \pm 0.02$ & $0.61 \pm 0.01$ & 0.99 \\
\hline 5 & $\mathrm{AOL}$ & Fuc a1-6GlcNAc (core Fuc), Fuc a1-2(Gal $\beta 1-4)$ GlcNAc & $0.93 \pm 0.34$ & $0.65 \pm 0.04$ & 1.43 & $0.58 \pm 0.04$ & $0.55 \pm 0.04$ & 1.05 \\
\hline 6 & AALa & $\begin{array}{l}\text { Fuc a1-6GlcNAc (core Fuc), Fuc a1-3(Gal } \beta 1-4) \text { GlcNAc } \\
\left(\text { Le }^{x}\right)\end{array}$ & $1.22 \pm 0.09$ & $1.07 \pm 0.04$ & $1.14^{*}$ & $0.95 \pm 0.05$ & $0.87 \pm 0.01$ & $1.10^{*}$ \\
\hline 7 & $M A L^{a}$ & Sia a2-3Gal $\beta 1-4 G \mid c N A c$ & $0.47 \pm 0.02$ & $0.60 \pm 0.01$ & $0.77^{* * *}$ & $0.63 \pm 0.02$ & $0.70 \pm 0.01$ & $0.90^{* *}$ \\
\hline 8 & SNA & Sia a2-6Gal/GalNAc & $0.82 \pm 0.02$ & $1.01 \pm 0.05$ & $0.81^{* *}$ & $1.03 \pm 0.03$ & $0.90 \pm 0.03$ & $1.14^{* *}$ \\
\hline 9 & SSA & Sia a2-6Gal/GalNAc & $0.75 \pm 0.04$ & $0.90 \pm 0.06$ & $0.84^{*}$ & $1.03 \pm 0.01$ & $0.92 \pm 0.01$ & $1.11^{* * *}$ \\
\hline 10 & TJA-I & Sia a2-6Gal/GalNAC & $1.65 \pm 0.01$ & $2.28 \pm 0.08$ & $0.73^{* * *}$ & $1.66 \pm 0.15$ & $1.56 \pm 0.06$ & 1.06 \\
\hline 11 & PHA-L & Tri/Tetra-antennary complex-type N-glycan & $0.45 \pm 0.03$ & $0.45 \pm 0.01$ & 1.00 & $0.54 \pm 0.01$ & $0.59 \pm 0.01$ & $0.91^{* *}$ \\
\hline 12 & ECA & Gal $\beta 1-4 G l c N A c$ & $0.86 \pm 0.04$ & $0.93 \pm 0.04$ & 0.93 & $1.12 \pm 0.04$ & $1.18 \pm 0.04$ & 0.95 \\
\hline 13 & RCA120 & Gal $\beta 1-4 \mathrm{GlcNAc}$ & $1.60 \pm 0.07$ & $2.43 \pm 0.20$ & $0.66^{* *}$ & $2.13 \pm 0.08$ & $2.25 \pm 0.12$ & 0.95 \\
\hline 14 & PHA-E & $\begin{array}{l}\text { Complex-type N-glycans with outer Gal and bisecting } \\
\text { GICNAC }\end{array}$ & $0.75 \pm 0.05$ & $1.33 \pm 0.06$ & $0.57^{* * *}$ & $1.01 \pm 0.02$ & $0.88 \pm 0.05$ & $1.15^{*}$ \\
\hline 15 & DSAa & $\begin{array}{l}(\text { GlcNAc } \beta 1-4)_{n} \text { Gal } \beta 1-4 G l c N A c \text {, Tri/Tetra-antennary } \\
\text { N-glycans }\end{array}$ & $1.75 \pm 0.03$ & $2.21 \pm 0.06$ & $0.79^{* * *}$ & $1.78 \pm 0.04$ & $2.04 \pm 0.04$ & $0.87^{* *}$ \\
\hline 16 & GSL-II & Agalactosylated tri/tetra antennary glycans, GlcNAc & $0.48 \pm 0.02$ & $0.31 \pm 0.00$ & $1.52^{* * *}$ & $0.44 \pm 0.02$ & $0.43 \pm 0.01$ & 1.04 \\
\hline 17 & NPA & High Man, Man a1-6Man & $1.52 \pm 0.17$ & $1.68 \pm 0.03$ & 0.90 & $1.41 \pm 0.04$ & $1.38 \pm 0.07$ & 1.02 \\
\hline 18 & ConA & $\begin{array}{l}\text { High Man, Man a1-6(Man a1-3)Man (inhibited by pres- } \\
\text { ence of bisecting GlcNAc) }\end{array}$ & $2.29 \pm 0.23$ & $2.23 \pm 0.16$ & 1.03 & $1.68 \pm 0.11$ & $1.59 \pm 0.09$ & 1.06 \\
\hline 19 & GNA & High Man, Man a1-3Man & $0.98 \pm 0.08$ & $1.10 \pm 0.01$ & 0.89 & $1.02 \pm 0.02$ & $0.97 \pm 0.03$ & 1.05 \\
\hline 20 & $\mathrm{HHL}$ & High Man, Man a1-3Man, Man a1-6Man & $0.67 \pm 0.04$ & $0.58 \pm 0.01$ & $1.17^{*}$ & $0.75 \pm 0.02$ & $0.74 \pm 0.03$ & 1.01 \\
\hline 21 & ACG & Sia a2-3Gal $\beta 1-4 G I c N A c$ & $1.36 \pm 0.18$ & $1.59 \pm 0.09$ & 0.85 & $1.38 \pm 0.07$ & $1.57 \pm 0.05$ & $0.88^{*}$ \\
\hline 22 & TXLC-I & $\begin{array}{l}\text { Man }_{3} \text { core, bi- and tri-antennary complex-type N-glycan, } \\
\text { GalNAc }\end{array}$ & $1.06 \pm 0.04$ & $0.80 \pm 0.05$ & $1.32^{* *}$ & $0.97 \pm 0.05$ & $0.93 \pm 0.04$ & 1.04 \\
\hline 23 & $\mathrm{BPL}$ & Gal $\beta 1-3$ GalNAc (a-Thr/Ser (T)), GalNAc & $0.54 \pm 0.05$ & $0.53 \pm 0.03$ & 1.01 & $0.66 \pm 0.02$ & $0.63 \pm 0.02$ & 1.05 \\
\hline 24 & TJA-II & $\begin{array}{l}\text { Fuc a1-2Gal } \beta 1-4 \text {, GalNAc } \beta 1-4 \text { groups at their nonreduc- } \\
\text { ing terminals }\end{array}$ & $0.92 \pm 0.04$ & $1.01 \pm 0.02$ & $0.90^{*}$ & $1.11 \pm 0.06$ & $1.12 \pm 0.03$ & 0.99 \\
\hline 25 & EEL & $\begin{array}{l}\text { Gal a1-3Gal } \beta 1-4 G \mid c N A c \text {, Fuc a1-2(Gal a 1-3)Galß1- } \\
\text { 4GIcNAc }\end{array}$ & $0.58 \pm 0.04$ & $0.33 \pm 0.02$ & $1.74^{* *}$ & $0.51 \pm 0.02$ & $0.52 \pm 0.01$ & 0.98 \\
\hline 26 & $\mathrm{ABA}^{\mathrm{a}}$ & Gal ß1-3GalNAc (a-Thr/Ser (T)), GlcNAc, sialyl-T & $2.16 \pm 0.08$ & $0.84 \pm 0.06$ & $2.58^{* * *}$ & $0.89 \pm 0.01$ & $0.84 \pm 0.01$ & $1.06^{*}$ \\
\hline 27 & LELa & $(\text { GlcNAc } \beta 1-4)_{n},(\text { Gal } \beta 1-4 G \mid c N A c)_{n}$ (polyLacNAc) & $2.39 \pm 0.12$ & $2.75 \pm 0.16$ & $0.87^{*}$ & $2.95 \pm 0.08$ & $3.16 \pm 0.09$ & $0.94^{*}$ \\
\hline 28 & STL & $\begin{array}{l}(\mathrm{GlcNAc})_{n^{\prime}}(\mathrm{GlcNAc} \beta 1-4 \mathrm{MurNAc})_{n} \text { (peptidoglycan } \\
\text { backbone) }\end{array}$ & $1.77 \pm 0.13$ & $2.19 \pm 0.14$ & $0.81^{*}$ & $2.21 \pm 0.16$ & $2.36 \pm 0.09$ & 0.94 \\
\hline 29 & UDA & GlcNAc $\beta 1-4 G l c N A c$, Mixture of Man $_{5}$ to Man $_{9}$ & $1.65 \pm 0.16$ & $2.09 \pm 0.32$ & 0.79 & $1.62 \pm 0.21$ & $1.94 \pm 0.26$ & 0.84 \\
\hline 30 & PWM & $(G \mid c N A c \text { a } 1-4)_{n}$ & $0.52 \pm 0.04$ & $0.58 \pm 0.08$ & 0.90 & $0.82 \pm 0.00$ & $0.84 \pm 0.03$ & 0.98 \\
\hline 31 & Jacalin & Gal $\beta 1$-3GalNAc (a-Thr/Ser (T)), GalNAc (a-Thr/Ser (Tn)) & $1.51 \pm 0.25$ & $1.55 \pm 0.15$ & 0.97 & $1.64 \pm 0.04$ & $1.60 \pm 0.09$ & 1.02 \\
\hline 32 & PNA ${ }^{a}$ & Gal $\beta 1-3$ GalNAc (a-Thr/Ser (T)) & $0.68 \pm 0.09$ & $0.36 \pm 0.01$ & $1.86^{* *}$ & $0.49 \pm 0.01$ & $0.43 \pm 0.01$ & $1.14^{* *}$ \\
\hline 33 & WFA & $\begin{array}{l}\text { Terminal GalNAc (e.g. GalNAcß1-4GlcNAc), Galß1-3(-6) } \\
\text { GalNAc }\end{array}$ & $0.52 \pm 0.05$ & $0.51 \pm 0.02$ & 1.03 & $0.71 \pm 0.08$ & $0.66 \pm 0.06$ & 1.08 \\
\hline 34 & ACA & Gal ß1-3GalNAc (a-Thr/Ser (T)) & $2.20 \pm 0.15$ & $1.16 \pm 0.14$ & $1.90^{* * *}$ & $0.87 \pm 0.07$ & $0.97 \pm 0.05$ & 0.90 \\
\hline 35 & MPA & Gal $\beta 1$-3GalNAc (a-Thr/Ser (T)), GalNAc (a-Thr/Ser (Tn)) & $0.81 \pm 0.08$ & $0.50 \pm 0.02$ & $1.61^{* *}$ & $0.71 \pm 0.04$ & $0.66 \pm 0.04$ & 1.08 \\
\hline 36 & HPA & a-Linked terminal GalNAc & $0.89 \pm 0.04$ & $0.43 \pm 0.05$ & $2.08^{* * *}$ & $0.45 \pm 0.03$ & $0.45 \pm 0.02$ & 1.01 \\
\hline 37 & WAa $^{a}$ & a-linked terminal GalNAc (a-Thr/Ser (Tn), GalNAc a1-3Gal & $0.55 \pm 0.03$ & $0.44 \pm 0.02$ & $1.24^{* *}$ & $0.59 \pm 0.03$ & $0.43 \pm 0.00$ & $1.35^{* * *}$ \\
\hline 38 & DBA & $\begin{array}{l}\text { GalNAc a1-3GalNAc (Blood group A), GalNAc } \\
\text { a1-3GalNAc }\end{array}$ & $0.41 \pm 0.04$ & $0.34 \pm 0.01$ & $1.21^{*}$ & $0.46 \pm 0.01$ & $0.46 \pm 0.01$ & 1.00 \\
\hline 39 & SBA & a or $\beta$-linked terminal GalNAc, GalNAc a1-3Gal & $0.56 \pm 0.06$ & $0.51 \pm 0.05$ & 1.10 & $0.78 \pm 0.04$ & $0.66 \pm 0.02$ & $1.18^{* *}$ \\
\hline 40 & Calsepa ${ }^{a}$ & $\begin{array}{l}\text { High Man (Man } 2-6), N \text {-glycans including bisecting } \\
\text { GlcNAC }\end{array}$ & $0.79 \pm 0.05$ & $0.66 \pm 0.02$ & $1.21^{*}$ & $0.80 \pm 0.05$ & $0.67 \pm 0.02$ & $1.20^{*}$ \\
\hline
\end{tabular}


Table 1 continued

\begin{tabular}{|c|c|c|c|c|c|c|c|c|}
\hline No. & Lectin & Preferred glycan structure (terminal epitope) & $\mathrm{SP} 1 \pm \mathrm{SD}$ & $\mathrm{MP} 1 \pm \mathrm{SD}$ & SP1/MP1 & $\mathrm{SP} 2 \pm \mathrm{SD}$ & $\mathrm{MP2} \pm \mathrm{SD}$ & SP2/MP2 \\
\hline 41 & PTL-I & a-Linked terminal GalNAC & $0.48 \pm 0.03$ & $0.53 \pm 0.05$ & 0.92 & $0.59 \pm 0.04$ & $0.50 \pm 0.02$ & $1.17^{*}$ \\
\hline 42 & MAH & Sia a2-3Gal $\beta 1-3($ Sia a2-6) GalNAc & $0.37 \pm 0.03$ & $0.49 \pm 0.04$ & $0.76^{*}$ & $0.53 \pm 0.02$ & $0.50 \pm 0.02$ & 1.05 \\
\hline 43 & WGAa & $(G \mid c N A c \beta 1-4)_{n}$ NeuAc, multivalent Sia & $1.32 \pm 0.09$ & $1.52 \pm 0.07$ & $0.87^{*}$ & $1.39 \pm 0.02$ & $1.48 \pm 0.03$ & $0.93^{* *}$ \\
\hline 44 & GSL-IA 4 & a-GalNAc (a-Thr/Ser (Tn)) & $0.53 \pm 0.06$ & $0.43 \pm 0.03$ & 1.22 & $0.70 \pm 0.01$ & $0.63 \pm 0.02$ & $1.11^{* *}$ \\
\hline 45 & GSL-IB ${ }_{4}^{a}$ & a-Gal & $0.75 \pm 0.02$ & $0.55 \pm 0.03$ & $1.35^{* * *}$ & $0.69 \pm 0.01$ & $0.62 \pm 0.02$ & $1.11^{* *}$ \\
\hline
\end{tabular}

a Significantly changed in two experiments; ${ }^{*} p<0.05 ;{ }^{* *} p<0.01 ;{ }^{* * *} p<0.001$

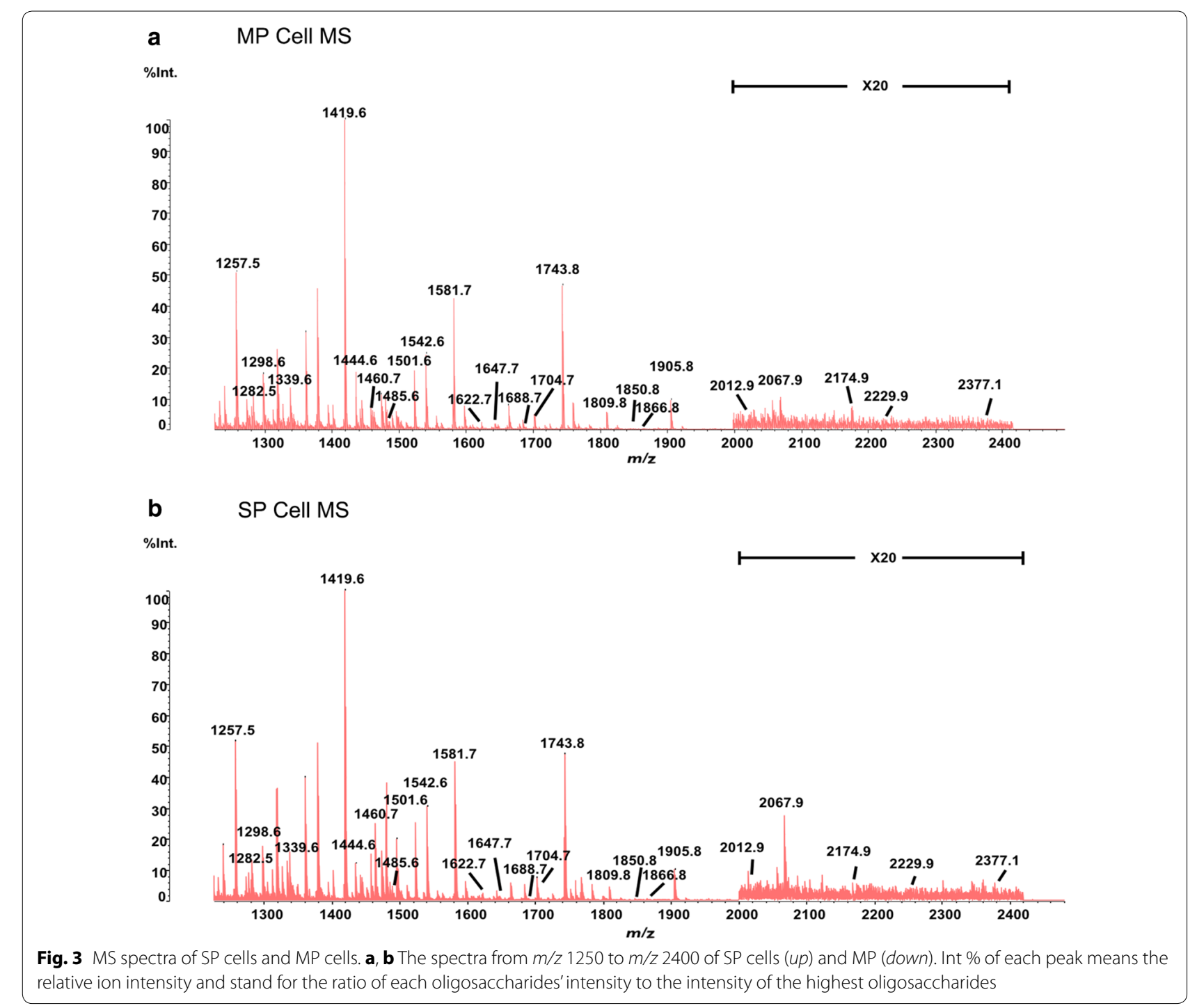

both lectins. For ABA, intensity of glycoprotein from 70-180 kDa was higher in SP cells. For VVA, glycoprotein from $50-80 \mathrm{kDa}$ showed stronger intensity in $\mathrm{SP}$ cells (Fig. 5a). These differences were further verified by real-time PCR. C1GALT1 (core 1 synthase, glycoprotein$\mathrm{N}$-acetylgalactosamine 3 -beta-galactosyltransferase 1 ), which acts on $\mathrm{Tn}$ to form a $\mathrm{T}$ antigen, was increased in SP cells at mRNA level. ST3Gal1 (ST3 beta-galactoside alpha-2,3-sialyltransferase 1), which can modify $\mathrm{T}$ antigens to form sT, was also enhanced in SP cells (Fig. 5b).

To investigate whether the SP cells continued to display enhanced binding for ABA and VVA during proliferation, 

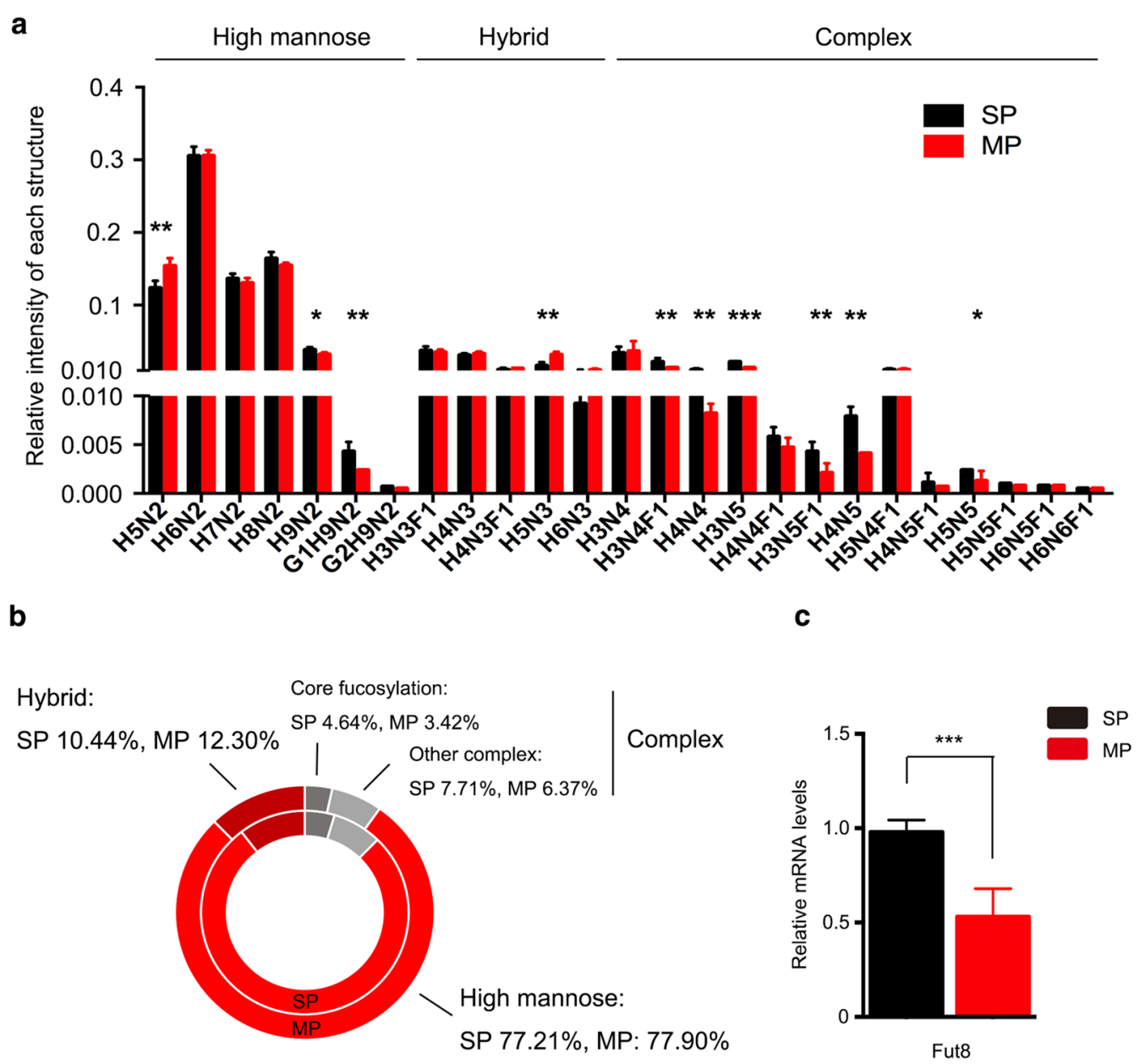

Fig. 4 Relative quantities of different classes of N-glycans in SP cells and MP cells. a Comparison of the relative quantities of each structure on SP cells and MP cell. The relative quantity of each structure was stand for the proportion of each structure relative intensity in total intensity of all 25 structures. b Comparison of the different classes of glycans using a pie chart: the inner circle represents SP cells, and the outer circle represents MP cells. c The expression of Fut8 was analysed by quantitative real-time RT-PCR

we detected the intensity of ABA and VVA for MP and SP sphere cells after 5 weeks' culture. Both ABA and VVA continued to strongly bind to SP cells, indicating that these lectins might be potential markers of ovarian cancer SP cells (Fig. 5c, d).

\section{Discussion}

Glycosylation plays important roles in many physiological and pathological processes as well as the identity and function of CSCs. However, the small population size of CSCs has hindered the characterization of glycans released from CSCs. Thus, we used lectin arrays to characterize the entire glycome of CSCs, both N-glycans and $\mathrm{O}$-glycans. Lectin array is time saving (1-day operation), requires small amount of cells (lower limit of 1000 cells). And it can analyze intact glycoproteins, which simplified sample preparation and made detection of sialic acid easy. We also used MS to characterize the structure of $\mathrm{N}$-glycans and provide information about the composition of the N-glycome. Combination of these two methods provided more comprehensive information about glycans in ovarian CSCs. In this study, we didn't conduct MS analysis of O-glycans, because it needs $10^{6}-10^{7}$ cells at least for analysis [24], and it was difficult to enrich enough HO8910 pm SP cells. New methods or technologies are still needed to overcome these obstacles.

Our present study suggested that SP cells may overexpress the tumor-associated antigens, $\mathrm{T}, \mathrm{Tn}$, and sT. The lectin arrays revealed enhanced intensity of VVA, SBA, and GSL-IA lectins in SP cells, which implied Tn antigen overexpession in SP cells. Increased expression of C1GALT1 and signal intensity of ABA and PNA lectins in 


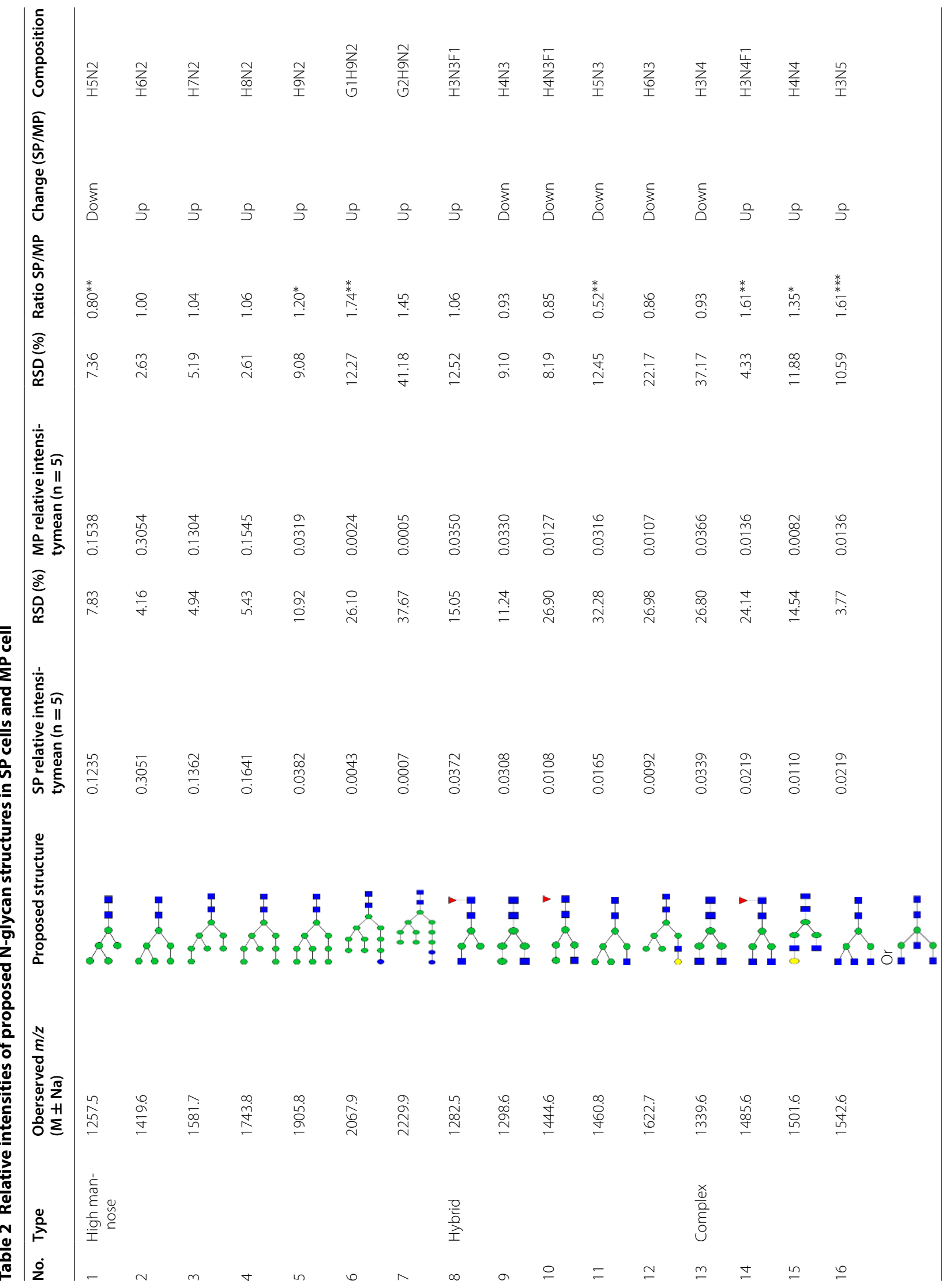




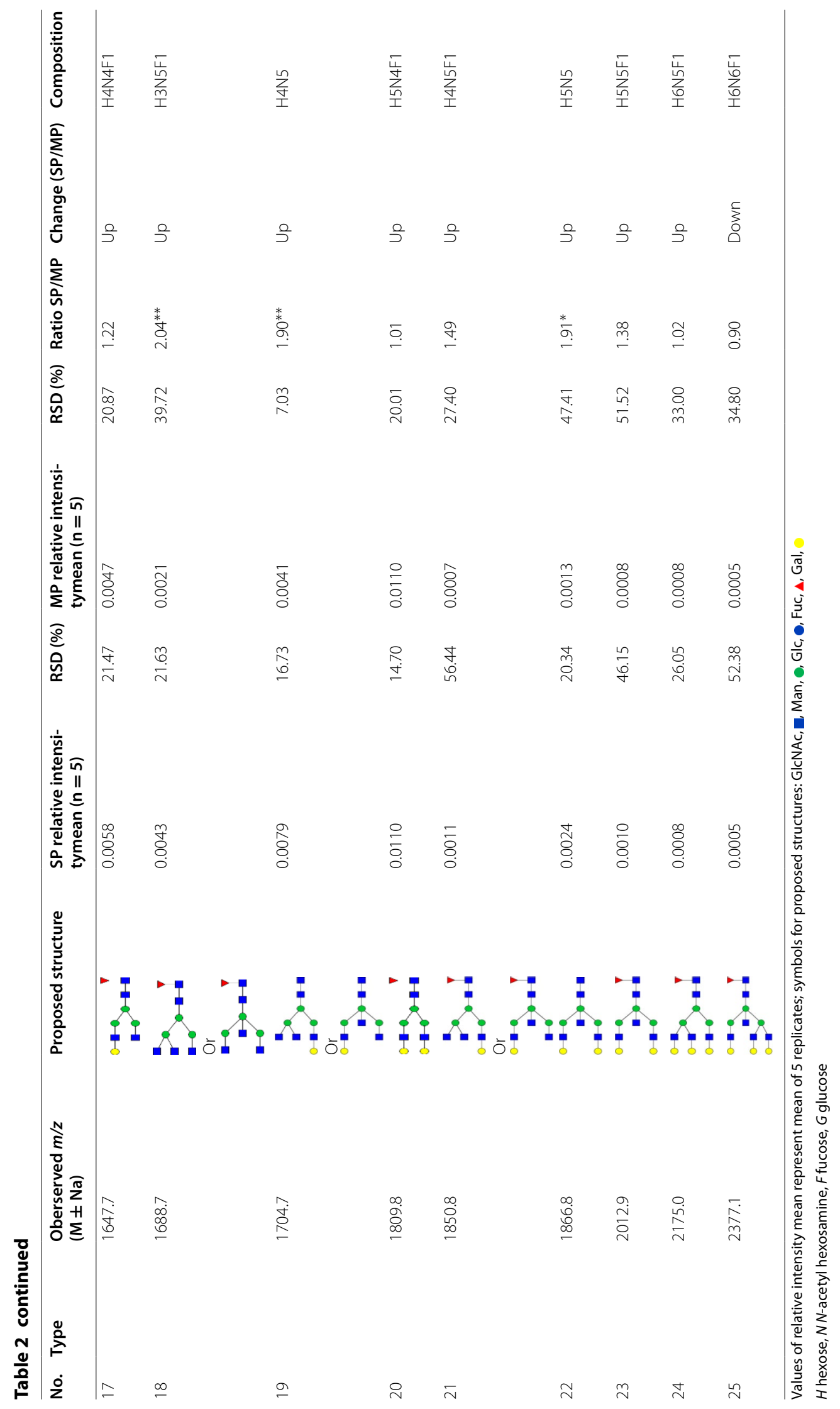




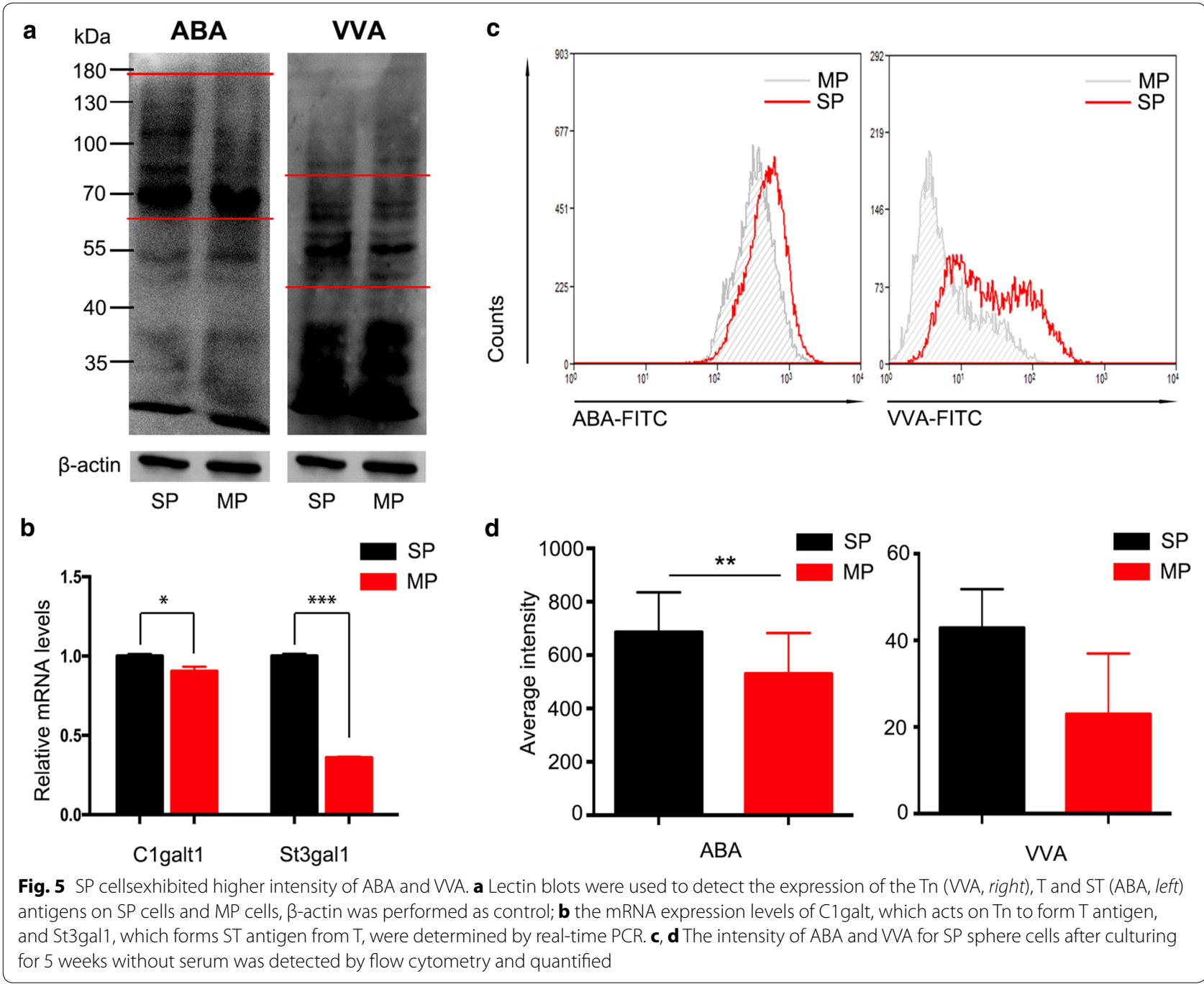

SP cells suggested upregulation of $\mathrm{T}$ antigen. Besides, $\mathrm{T}$ antigens were modified by ST3Gal1, which was enhanced in SP cells, to form sT antigen recognized by ABA lectin. Our lectin blot results also confirmed stronger intensity of ABA, VVA for SP cells. Furthermore, after 5 weeks' culture without serum, the SP sphere cells still displayed higher intensity of ABA and VVA compared with MP cells, indicating that the expression of these antigens continued during CSC proliferation. To further verify whether these changes were only HO8910 pm cell line specific, we used another ovarian cancer cell line ES-2 for SP cell sorting and validated that ES-2 SP cells also showed higher intensity of ABA and VVA than ES-2 MP cells (see Additional file 1).

In our MS analysis, glycans with terminal Glcs (glucose), which are usually related to glycoprotein quality control $[25,26]$ and exhibit higher expression in cancer [27, 28], were expressed more abundantly in SP cells. Increased hybrid N-glycans were detected in MP cells, indicating that the immediate products of GlcNAc transferase I were not further modified by other GlcNAc transferases. Among more mature N-glycans, core fucosylation $\mathrm{N}$-glycans were more abundant in SP cells, as verified by MS and lectin array. The mRNA transcript level of FUT8, which synthesises the core fucosylation, was also enhanced. Increased core fucose is closely associated with many types of cancer. It showed an unfavourable clinical outcome and played as a functional regulator of non-small cell lung cancer [29, 30]. Loss of FUT8 also down-regulates several cellular signaling pathways to inhibit chemical-induced hepatocellular carcinoma [31].

The common available biomarkers of ovarian CSCs are ALDH, CD133+, CD24+ CD44+, CD44+ CD117+ and negative Hoechst 33342 stain. The use of a single marker has disadvantages because not all cancer cell lines or tissues are positive for the marker and the cells enriched for 
a marker maybe not purified. In addition, ALDH, CD133, CD44, and CD177 are also expressed in normal stem cells. Thus, new biomarkers are still needed, and glycans may provide a breakthrough.

This study implied higher levels of Tn, T and sT antigens in ovarian cancer SP cells. Tn, T and sT antigens are weakly expressed in normal tissues but overexpressed in most human carcinomas and are associated with poor prognosis [32-34]. A recent review by Karsten and Goletz [19] hypothesized that $\mathrm{T}$ antigen might be a potential biomarker of CSCs. Because this antigen is only expressed in onco-foetal stem cells and tumor cells, targeting $\mathrm{T}$ antigen should not harm normal adult stem cells. Lin et al. also confirmed the co-expression of $\mathrm{T}$ antigen with the stem cell markers CD44 and CD133 in lung, breast and liver cancer [35].

Our results are similar to those of previous lectin studies of CSCs. In glioblastoma, lectins recognizing GalNAc and GlcNAc exhibit higher affinity for the surface of undifferentiated cells, suggesting that GalNAc and GlcNAc might be a novel biomarker to identify CSCs [19]. In the same study, the lectins PNA, GSL-I, SBA, and VVA also exhibited higher signals in CSCs. In pancreatic CSCs, Terao et al. observed enhanced ABA and VVA intensity of CSCs using the same lectin array [36]. In addition, liver $\mathrm{CSC}$ isolated as $\mathrm{CD} 133^{+} \mathrm{CD} 13^{+}$cells also exhibited stronger intensities of SNA, SSA, ABA, PNA, and SBA [18].

Based on the results of the present study and previous reports, we hypothesize that tumor-associated glycan antigens may be novel potential CSC markers. Future studies should examine whether lectin ABA combined with available markers can isolate more malignant CSCs.

\section{Conclusions}

In conclusion, we conducted a systematic glycomic analysis of ovarian cancer SP cells using lectin arrays and MS. Our findings suggested that SP cells expressed increased core fucose, Tn, $\mathrm{T}$ and $\mathrm{sT}$ antigens. In addition, hybrid type, $\alpha 2,3$-linked sialic glycan, and multivalent sialyl-glycan were enhanced in MP cells. Aberrant glycan profiles may be related to some stemness properties of SP cells and may provide potential biomarkers for ovarian cancer CSCs (all lectins in the array: see Additional file 2).

\section{Additional files}

Additional file 1. SP cells sorted from ES-2 cells exhibited higher intensity of ABA and WVA. The intensity of ABA (left) and WA (right) for SP sphere cells after culturing for 1 weeks without serum was detected by flow cytometry.

Additional file 2. The category of representative lectins for glycan profiling.

\section{Abbreviations}

CSCs: cancer stem cells; SP: side population; MP: main population; PI: propidium iodide; MS: mass spectrometry; Fuc: fucose; Gal: galactose; GalNAc: $\mathrm{N}$-acetylgalactosamine; Glc: glucose; ACN: acetonitrile; TFA: trifluoroacetic acid; PBS: phosphate-buffered saline; SDS-PAGE: sodium dodecyl sulfatepolyacrylamide gel electrophoresis; PVDF: polyvinylidene difluoride; ABA: Agaricusbisporus; VA: Viciavillosa; PCR: polymerase chain reaction; SSA: Sambucussieboldiana; FUT8: fucosyltransferase-8; C1 GALT1: core1 synthase, glycoprotein- $N$-acetylgalactosamine 3-beta-galactosyltransferase 1; ST3Gal1: ST3 beta-galactoside alpha-2,3-sialyltransferase 1; Tn antigen: GalNAc a-Ser/ Thr; T antigen: Galß1-3GalNAc a-Ser/Thr (Thomsen-Friedenreich antigen); sT antigen: sia a2-3Gal $\beta 1$-3GalNAc a-Ser/Thr (sialyl-T).

\section{Authors' contributions}

RZ performed the experiments, analyzed the data, and drafted the manuscript. XXL participated in the study design, analyzed the data, and drafted the manuscript. SFR, CJX and YSW participated in the study design, data analyzing, and drafted the manuscript. YYR and JXG participated in the design of this study. XXJ, RHQ, WJQ and HW performed the experiments and analyzed the data. MYZ and HYT contributed to the research materials. CTY, LLL, PKP, and MMS participated in the experiments and analyzed the data. All authors read and approved the final manuscript.

\section{Author details}

${ }^{1}$ Institute of Biomedical Sciences, Fudan University, 138 Yi-Xueyuan Road, Shanghai 200032, People's Republic of China. ${ }^{2}$ Obstetrics and Gynecology Hospital of Fudan University, 419 Fang-Xie Road, Shanghai 200011, People's Republic of China. ${ }^{3}$ Key Laboratory of Glycoconjugate Research Ministry of Public Health, Department of Biochemistry and Molecular Biology, School of Basic Medical Sciences, Fudan University, 138 Yi-Xueyuan Road, Shanghai 200032, People's Republic of China. ${ }^{4}$ Department of Obstetrics and Gynecology of Shanghai Medical School, Fudan University, Shanghai 200032, People's Republic of China. ${ }^{5}$ Shanghai Key Laboratory of Female Reproductive Endocrine Related Diseases, Shanghai 200032, People's Republic of China.

\section{Acknowledgements}

We acknowledge Jing Qian and Yuting Deng from the department of Immunology, Fudan University, for providing MoFlo XDP cytometer.

\section{Competing interests}

The authors declare that they have no competing interests.

\section{Availability of data and material}

The datasets supporting the conclusions of this article are included within the article.

\section{Funding}

This work was supported by National Key Research and Development Program (2016YFA0501303, 2016YFC1303102), National Natural Sciences Foundation of China $(81272879,81302258,81572352)$, the National Basic Research Program of China (973 Program; 2012CB8221004 and 2013CB910503).

Received: 15 July 2016 Accepted: 24 September 2016

Published online: 10 November 2016

References

1. Siegel RL, Miller KD, Jemal A. Cancer statistics, 2016. CA Cancer J Clin. 2016;66:7-30.

2. Partridge EE, Barnes $M N$. Epithelial ovarian cancer: prevention, diagnosis, and treatment. CA Cancer J Clin. 1999;49:297-320.

3. Li J, Fadare O, Xiang L, Kong B, Zheng W. Ovarian serous carcinoma: recent concepts on its origin and carcinogenesis. J Hematol Oncol. 2012;5:8.

4. Lengyel E. Ovarian cancer development and metastasis. Am J Pathol. 2010;177:1053-64.

5. Poveda A, Romero I. Advanced ovarian cancer: 20 years of ovarian cancer treatment. Ann Oncol. 2016;27(Suppl 1):i72-3. 
6. Tomao F, Papa A, Rossi L, Strudel M, Vici P, Lo Russo G, et al. Emerging role of cancer stem cells in the biology and treatment of ovarian cancer: basic knowledge and therapeutic possibilities for an innovative approach. J Exp Clin Cancer Res. 2013;32:48.

7. Ffrench B, Gasch C, O'Leary JJ, Gallagher MF. Developing ovarian cancer stem cell models: laying the pipeline from discovery to clinical intervention. Mol Cancer. 2014;13:262.

8. Kwon MJ, Shin YK. Regulation of ovarian cancer stem cells or tumorinitiating cells. Int J Mol Sci. 2013;14:6624-48.

9. Schulenburg A, Blatt K, Cerny-Reiterer S, Sadovnik I, Herrmann H, Marian $B$, et al. Cancer stem cells in basic science and in translational oncology: can we translate into clinical application? J Hematol Oncol. 2015;8:16.

10. Abbott KL, Lim JM, Wells L, Benigno BB, McDonald JF, Pierce M. Identification of candidate biomarkers with cancer-specific glycosylation in the tissue and serum of endometrioid ovarian cancer patients by glycoproteomic analysis. Proteomics. 2010;10:470-81.

11. Xu Q, Akama R, Isaji T, Lu Y, Hashimoto H, Kariya Y, et al. Wnt/beta-catenin signaling down-regulates $\mathrm{N}$-acetylglucosaminyltransferase III expression: the implications of two mutually exclusive pathways for regulation. J Biol Chem. 2011;286:4310-8.

12. Abbott KL, Nairn AV, Hall EM, Horton MB, McDonald JF, Moremen KW, et al. Focused glycomic analysis of the N-linked glycan biosynthetic pathway in ovarian cancer. Proteomics. 2008;8:3210-20.

13. Li S, Mo C, Peng Q, Kang X, Sun C, Jiang K, et al. Cell surface glycan alterations in epithelial mesenchymal transition process of Huh7 hepatocellular carcinoma cell. PLOS ONE. 2013;8:e71273.

14. Rachagani S, Torres MP, Kumar S, Haridas D, Baine M, Macha MA, et al. Mucin (Muc) expression during pancreatic cancer progression in spontaneous mouse model: potential implications for diagnosis and therapy. J Hematol Oncol. 2012;5:68.

15. Croci DO, Cerliani JP, Dalotto-Moreno T, Mendez-Huergo SP, Mascanfroni ID, Dergan-Dylon S, et al. Glycosylation-dependent lectin-receptor interactions preserve angiogenesis in anti-VEGF refractory tumors. Cell. 2014;156:744-58.

16. Desiderio V, Papagerakis P, Tirino V, Zheng L, Matossian M, Prince ME, et al. Increased fucosylation has a pivotal role in invasive and metastatic properties of head and neck cancer stem cells. Oncotarget. 2015;6:71-84.

17. Che MI, Huang J, Hung JS, Lin YC, Huang MJ, Lai HS, et al. Beta1, 4-N-acetylgalactosaminyltransferase III modulates cancer stemness through EGFR signaling pathway in colon cancer cells. Oncotarget. 2014;5:3673-84.

18. Moriwaki K, Okudo K, Haraguchi N, Takeishi S, Sawaki H, Narimatsu H, et al. Combination use of anti-CD133 antibody and SSA lectin can effectively enrich cells with high tumorigenicity. Cancer Sci. 2011;102:1164-70.

19. Karsten U, Goletz S. What makes cancer stem cell markers different? Springerplus. 2013;2:301.

20. Jiang H, Lin X, Liu Y, Gong W, Ma X, Yu Y, et al. Transformation of epithelial ovarian cancer stemlike cells into mesenchymal lineage via EMT results in cellular heterogeneity and supports tumor engraftment. Mol Med. 2012;18:1197-208.
21. Zhang X, Wang Y, Qian Y, Wu X, Zhang Z, Liu X, et al. Discovery of specific metastasis-related $\mathrm{N}$-glycan alterations in epithelial ovarian cancer based on quantitative glycomics. PLoS ONE. 2014;9:e87978.

22. Tan Z, Lu W, Li X, Yang G, Guo J, Yu H, et al. Altered N-glycan expression profile in epithelial-to-mesenchymal transition of NMuMG cells revealed by an integrated strategy using mass spectrometry and glycogene and lectin microarray analysis. J Proteome Res. 2014;13:2783-95.

23. Reiding KR, Blank D, Kuijper DM, Deelder AM, Wuhrer M. High-throughput profiling of protein N-glycosylation by MALDI-TOF-MS employing linkage-specific sialic acid esterification. Anal Chem. 2014;86:5784-93.

24. Furukawa J, Piao J, Yoshida Y, Okada K, Yokota I, Higashino K, et al. Quantitative O-glycomics by microwave-assisted beta-elimination in the presence of pyrazolone analogues. Anal Chem. 2015;87:7524-8.

25. Chen Y, Hu D, Yabe R, Tateno H, Qin SY, Matsumoto N, et al. Role of malectin in Glc(2)Man(9)GlcNAc(2)-dependent quality control of alpha1antitrypsin. Mol Biol Cell. 2011;22:3559-70.

26. Fanchiotti S, Fernandez F, D'Alessio C, Parodi AJ. The UDP-Glc: glycoprotein glucosyltransferase is essential for Schizosaccharomyces pombe viability under conditions of extreme endoplasmic reticulum stress. J Cell Biol. 1998;143:625-35.

27. Amin A, Bashir A, Zaki N, McCarthy D, Ahmed S, Lotfy M. Insights into glycan biosynthesis in chemically-induced hepatocellular carcinoma in rats: a glycomic analysis. World J Gastroenterol. 2015;21:6167-79.

28. Anugraham M, Jacob F, Nixdorf S, Everest-Dass AV, Heinzelmann-Schwarz $\mathrm{V}$, Packer $\mathrm{NH}$. Specific glycosylation of membrane proteins in epithelial ovarian cancer cell lines: glycan structures reflect gene expression and DNA methylation status. Mol Cell Proteom. 2014;13:2213-32.

29. Chen $C Y$, Jan $Y H$, Juan $Y H$, Yang CJ, Huang MS, Yu CJ, et al. Fucosyltransferase 8 as a functional regulator of nonsmall cell lung cancer. Proc Natl Acad Sci USA. 2013;110:630-5.

30. Honma R, Kinoshita I, Miyoshi E, Tomaru U, Matsuno Y, Shimizu Y, et al. Expression of fucosyltransferase 8 is associated with an unfavorable clinical outcome in non-small cell lung cancers. Oncology. 2015;88:298-308.

31. Wang Y, Fukuda T, Isaji T, Lu J, Im S, Hang Q, et al. Loss of alpha1,6-fucosyltransferase inhibits chemical-induced hepatocellular carcinoma and tumorigenesis by down-regulating several cell signaling pathways. FASEB J. 2015;29:3217-27.

32. Julien S, Videira PA, Delannoy P. Sialyl-tn in cancer: (how) did we miss the target? Biomolecules. 2012;2:435-66.

33. Munkley J. The role of sialyl-Tn in cancer. Int J Mol Sci. 2016;17:275.

34. Videira PA, Correia M, Malagolini N, Crespo HJ, Ligeiro D, Calais FM, et al. ST3Gal. I sialyltransferase relevance in bladder cancer tissues and cell lines. BMC Cancer. 2009;9:357.

35. Lin WM, Karsten U, Goletz S, Cheng RC, Cao Y. Expression of CD176 (Thomsen-Friedenreich antigen) on lung, breast and liver cancer-initiating cells. Int J Exp Pathol. 2011;92:97-105.

36. Terao N, Takamatsu S, Minehira T, Sobajima T, Nakayama K, Kamada Y, et al. Fucosylation is a common glycosylation type in pancreatic cancer stem cell-like phenotypes. World J Gastroenterol. 2015;21:3876-87.

\section{Submit your next manuscript to BioMed Central and we will help you at every step:}

- We accept pre-submission inquiries

- Our selector tool helps you to find the most relevant journal

- We provide round the clock customer support

- Convenient online submission

- Thorough peer review

- Inclusion in PubMed and all major indexing services

- Maximum visibility for your research

Submit your manuscript at www.biomedcentral.com/submit 